\title{
QoS-aware Resource Allocation in Relay-enhanced TD-LTE-A Systems
}

\author{
Yujie Pei ${ }^{1, a}$, Liqiang Wang ${ }^{2, b}$, Xuanli $\mathrm{Wu}^{1, \mathrm{c}}$ \\ ${ }^{1}$ Communication Research Center, Harbin Institute of Technology, Harbin, China \\ ${ }^{2}$ China Academy of Launch Vehicle Technology R\&D Center, Beijing, China \\ aemail: peiyujie1995@163.com, bemail: liqiangwang@163.com, email: xlwu2002@hit.edu.cn
}

\begin{abstract}
Keywords: TD-LTE-A; Relay; QoS-aware; Resource Allocation; SPRC;
\end{abstract}
\begin{abstract}
As a result of the introduction of relay technique in the Long Term Evolution Advanced (LTE-A) system, the system performance is improved in network coverage, user (UE) fairness and system throughput. Nevertheless, it is important to support the transmission of multimedia application and meet various quality-of-service (QoS) requirements of different traffics. Thus, a two-hop relay system scheduling several QoS-promising traffics is set and we propose a resource allocation algorithm named SPRC. The superiority of the proposed resource allocation algorithm is examined through system level simulation, compared with the two existing algorithms: TH-MLWDF and TFQoS. The results show that PLR is lower, GBR demand is well satisfied, fairness between R-UEs and D-UEs is guaranteed and system throughput is improved by SPRC.
\end{abstract}

\section{Introduction}

The Third Generation Partnership Project (3GPP) puts forward the relay technique to improve system performance in relay-enhanced TD-LTE-A systems [1]. Actually, not only the performance of system throughput and UE fairness are required to improve, but also various QoS requirements of UEs calls for gratification. It is essential to provide more impeccable service to UEs.

In recent years, many researchers are focusing on the study of the resource allocation scheme in wireless relay networks. The authors in [2] used a proportional fairness (PF) utility as the objective function. However, the demand of various QoS requirements is not considered. A low-complexity QoS-aware resource allocation algorithm aiming at maximizing system throughput is proposed in [3] and [4], but they do not consider the effect of delay, which is an influential factor to guarantee QoS. The authors in [5] adopt a two-stage scheduling algorithm at both the evolved NodeB (eNB) and Relay Nodes (RNs). The higher stage scheduling considers the delay demand, while the lower one employs the PF algorithm. However, it does not take the resource division between the eNB and RNs into account, i.e., all UEs are R-UEs. Ref. [6] shows that QoS-relevant parameters have an influence on the capacity in relay networks theoretically, and the simulation shows that maximum capacity is reduced with the growth of the required data rate of UEs, while advanced with the growth of the allowed delay. The authors in [7] propose a QoS-aware scheduling policy with the goal of achieving balance of traffic delay and bit rate.

Aiming at solving the problems in these existing papers, this paper proposes a QoS-guaranteed resource scheduling algorithm, which brings data rate, time delay and the service priority factors into the scheduling priority formula in TD-LTE-A uplink system with RNs. In LTE uplink, we should reduce the Peak to Average Power Ratio (PAPR) concerning terminal equipment cost, hence Single-carrier Frequency-Division Multiple Access (SC-FDMA) is employed. In order to ensure the orthogonality of the subcarriers in uplink, the subcarriers assigned to each UE must be continuous in the frequency domain, which should be considered in the scheduling algorithm.

\section{System Model}

\section{A. Communication Scenario in TD-LTE-A Relay System}

In this paper, in-band relays are adopted, which multiplex the same frequency resources in access and backhaul links. And the relay is time division half-duplex. What's more, DF protocol is 
employed in this paper.

Fig.1 shows the communication scenario in which various QoS-aware traffics indicated by the color bars in Fig. 1 are scheduled. In the model, UEs transfer information in the form of data packet, and the eNB as well as RNs are equipped with a fixed size First In First Out (FIFO) register respectively. There are two kinds of UEs and three kinds of transmission links in the system. UEs possess preferable channel condition to the eNB are D-UEs, which can communicate with the eNB directly. While UEs move far away from the eNB and experience poorer channel condition are R-UEs, which communicate with the eNB through a RN. For R-UEs, the link which connects the eNB and its serving RN is backhaul link L1, and the access link L2 connects the RN and R-UEs. For D-UEs, the link which connects to the eNB directly is direct link L3.

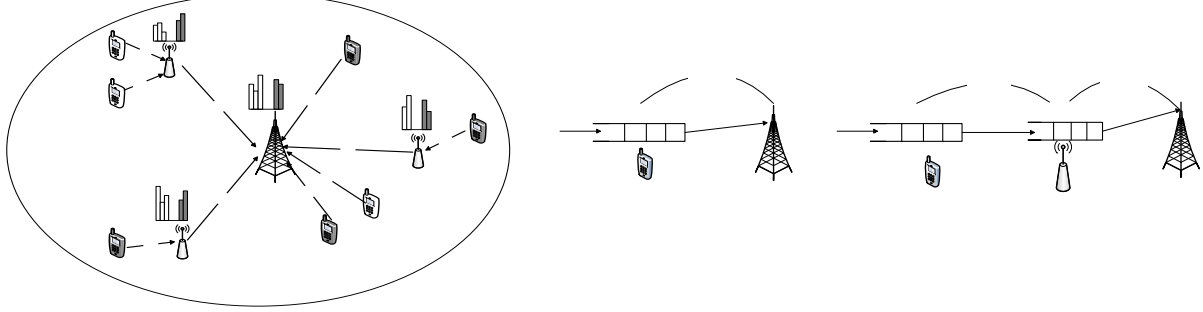

Fig.1 QoS-aware TD-LTE-A system model
Fig.2 Queue model in relay network

Fig. 3 Target delays in two-hop connection

\section{B. QoS in Relay Scenario}

The data queue model of QoS-promising traffics in relay scenario is indicated in Fig.2. The access delay of D-UEs is mainly derived from the waiting time of data packet. Providing that the traffic type of a D-UE is traffic $i$, then the delay of data packet and data rate are requested to meet:

$$
\begin{aligned}
& T_{U E-e N B}<\operatorname{Delay}(i) \\
& R_{U E-e N B}>G B R(i)
\end{aligned}
$$

Where, $T_{U E-e N B}$ is the transmission interval of data packets from the D-UE to eNB. Delay $(i)$ is the allowable maximum delay for traffic $i . R_{U E-e N B}$ is the actual transmission rate of this UE in direct links, and $G B R(i)$ is the minimum transmission rate that traffic $i$ should ensure.

For R-UEs, the QoS-aware resource scheduling experiences two phases. In the first stage, R-UEs send its data packet to its serving $\mathrm{RN}$ through access links. In the second stage, each RN sends the data packet from its buffer queue to the eNB. In other words, the transmission of R-UEs' data packet goes through two scheduling steps, which brings in extra delay. Hence, we define the QoS requirements of R-UEs as follows:

$$
T_{U E-R N}+T_{R N-e N B}<\operatorname{Delay}(i)
$$

$$
\frac{B_{R U E}}{T_{U E-R N}+T_{R N-e N B}}>G B R(i)
$$

Where, $T_{U E-R N}$ is the transmission interval of data packet from the R-UE to its serving RN. $T_{R N-e N B}$ is the transmission interval of data packets from the RN to eNB. Similarly, Delay(i) is the allowable maximum delay for traffic $i$. Moreover, $B_{R U E}$ is the data volume transmitted during $T_{U E-R N}+T_{R N-e N B}, G B R(i)$ is the minimum transmission rate that traffic $i$ need to guarantee.

\section{QoS-aware Resource Allocation Scheme}

The difference of QoS-aware resource scheduling between relay system and normal cellular system lies in the existence of R-UEs. We divide the QoS-aware resource allocation scheme in TD-LTE-A relay system into two steps: scheduling in backhaul sub-frame and scheduling in access and direct sub-frame. We will introduce the proposed SPRC algorithm in detail, compared with Two-hop Maximum-Largest Weighted Delay First (TH-MLWDF) and Time Domain and Frequency Domain QoS-aware scheduling algorithm (TFQoS) below.

\section{A. TH-MLWDF Algorithm}

Based on the classical MLWDF algorithm ${ }^{[8]}$, which possesses great performance in system 
throughput, Packet Loss Rate (PLR) and UE fairness. A UE is scheduled if:

$u e_{-} k=\underset{k}{\arg \max } a_{k} W_{k}(t) \frac{R_{k}(t)}{\bar{R}_{k}(t)}$

Where, $a_{k}=-\frac{\log \delta_{k}}{\tau_{k}}$, and $W_{k}(t)$ denotes Head Of Line (HOL) packet delay of UE $k$ at time $t$, $\tau_{k}$ is the preset time delay threshold of UE $k, \delta_{k}$ is the maximum probability of HOL packet to discard. And $R_{k}(t)$ is momentary achievable data rate of UE $k$ at time $t$, while $\bar{R}_{k}(t)$ denotes average data rate in time window.

TH-MLWDF algorithm is proposed in [9] to meet the QoS demands of various traffics in two-hop relay system. With the presence of RNs in the model, delay is divided into two parts as expressed in the formula (3), also shown in Fig. 3, where $D_{U E-e N B}$ is the allowable maximum delay for traffic $i$ from UE to eNB, the same as Delay(i) defined in (1). $D_{U E-R N}$ and $D_{R N-e N B}$ are preset delay threshold for traffic $i$ from UE to its serving RN and from RN to the eNB, respectively. In [9], the target delays in two-hop links are set as follows:

$$
D_{U E-R N}=D_{R N-e N B}=D_{U E-e N B} / 2
$$

\section{B. TFQoS Algorithm}

In TFQoS algorithm ${ }^{[10]}$, the scheduling process splits in two-stage: Time Domain (TD) scheduler and Frequency Domain (FD) scheduler. TD scheduler makes the UEs with traffics to be transmitted into a line. And selects UEs with higher priority into the candidate set. The priority formula is:

$$
Q_{k, Q Q S-T D}(t)= \begin{cases}\max \left[\left(\frac{G B R_{k}}{\bar{R}_{k}(t)}\right), 1\right]^{\rho} \cdot \frac{\omega_{k, d}(t)}{P(n)}, G B R \\ \frac{\omega_{k, d}(t)}{P(n)} & \text { Non-GBR }\end{cases}
$$

Where, $\omega_{k, d}(t)=\exp \left(\beta \frac{H O L_{k}(t)}{\text { Delay }_{k}}\right)$.

The candidate set of UEs formed by TD scheduler is sent to the FD scheduler, which allocates $\mathrm{RB}$ for the selected UEs. Then update priority matrix and repeat this procedure until RB set or candidate UEs set is empty. Besides, the priority formula is expressed as:

$$
Q_{k, Q S S-F D}(t)=\max \left[\left(\frac{G B R_{k} \cdot r_{k, n}(t)}{\bar{R}_{k}(t)}\right), 1\right]^{\rho} \cdot \frac{\omega_{k, d}(t)}{P(n)}
$$

Where, $\beta=5, \rho=4$.

\section{The proposed SPRC Algorithm}

Sub-problem 1: Access and direct Subframe Scheduling

In the access sub-frames, D-UEs compete with R-UEs for system resources. Therefore, we should set scheduling priority for both D-UEs and R-UEs. For all the real-time traffics, the designed priority formula should consider the delay constraint. The priority in our design takes data rate, time delay as well as the service priority ${ }^{[11]}$ into account, so we give the formula of priorities as below:

$$
Q_{k, n}(t)=\left\{\begin{array}{l}
\log _{10}\left(G B R_{k}\right) \cdot \frac{H O L_{k}(t)}{\text { Delay }_{k}} \cdot \frac{r_{k, n}(t)}{\overline{\mathrm{R}}_{k}(t)} \cdot(10-P(n)), G B R \\
\frac{H O L_{k}(t)}{\text { Delay }_{k}} \cdot \frac{r_{k_{n}, n}(t)}{\mathrm{R}_{k}(t)} \cdot(10-P(n)), \quad \quad \text { Non-GBR }
\end{array}\right.
$$

Where, $G B R_{k}$ is the minimum transmission rate to be guaranteed of UE $k . H O L_{k}$ is the data packet delay of the HOL of UE $k$, and Delay $k$ is the waiting time delay threshold of UE $k$. When the waiting time delay of a data packet exceeds the threshold, the data packet will be discarded. The closer is the HOL delay to the threshold, the higher the priority is, and the packets with the maximum priority will be scheduled preferentially; $P(n)$ is the service priority of UE $k$ with the value range 1-9. UEs with a smaller service priority value have a higher priority. $r_{k, n}(t) / \overline{\mathrm{R}}_{k}(t)$ is PF factor in scheduling algorithm to realize compromise between fairness and throughput.

For D-UEs or R-UEs, the criteria to schedule RB $n$ at time $t$ is:

$u e_{-} k=\underset{k}{\arg \max } Q_{k, n}(t)$ 
In particular, there are several matters need to take our attention in the priority formula about R-UEs. The transmission of R-UEs needs to jump through two links, so we need to redefine the QoS requirements of R-UEs in access link. If the maximum allowable delay of D-UEs for traffic $i$ is $\operatorname{delay}(i)$, the maximum allowable delay of R-UEs with the same traffic $i$ can be defined as:

$\operatorname{Delay}_{k, U E-R N}=\operatorname{Delay}(i) / 2$

If the GBR of D-UEs with traffic $i$ is expressed as GBR(i), the GBR of R-UEs with the same traffic $i$ in access links can be defined as:

$G B R_{\text {TTI,UE-RN }}=2 \cdot G B R_{\text {TTI }}(i)$

Therefore, when other conditions are fixed, for R-UEs and D-UEs with the same QoS requirements, the scheduling priority of R-UEs is higher.

The scheduling algorithm can be achieved through steps as follows:

Step 1: We can decide whether a UE is D-UE or R-UE according to the access to the eNB or RN standards. List all the services of D-UEs and R-UEs. Initialize the RB set as $N=\{1,2, . ., n, . ., N\}$ and UE queue information at the eNB, RB set $R B_{D}$ of D-UEs and RB set $R B r$ of R-UEs belong to $\mathrm{RN} r$.

Step 2: $n=1$, namely, the RB allocation begins from the first RB.

Step 3: According to the CQI in direct links reported by D-UEs, the eNB calculates the bit rate of each D-UE on RB $n$ in theory. Similarly, the RN $r$ calculates the bit rate of its relevant R-UEs on $\mathrm{RB} n$ in theory, based on the CQI in access links reported by R-UEs.

Step 4: According to the priority formula and the outputs of Step3, the priority values of each UE on its corresponding RB $n$ at the current TTI can be calculated. From the service lists, we can find the UE $k$ possessing the highest priority and schedule it on $\mathrm{RB} n$, then delete it from RB set. If UE $k$ is D-UEs, we get $R B_{D}=R B_{D} \cup\{n\}$. And if UE $k$ is R-UEs and belongs to RN $r$, we get $R B_{r}=R B_{r} \cup\{n\}$.

Step 5: Update the current queue information of UE $k$. If the queue of UE $k$ is empty or the obtained data rate of UE $k$ in the current TTI is greater than the GBR requirements, remove the UE from the services list. Otherwise, the UE remains in the services list to be scheduled.

Step 6: $n=n+1$, remove the RB $n$ from the RB set, repeat steps 3 to 6 until $N=\varnothing$ or the services list is empty.

\section{Sub-problem 2: Backhaul Subframe Scheduling}

Due to the characteristic of in-band relay, in the backhaul frame, eNB allocates RBs from set $R B_{D}$ to D-UEs to meet its QoS demands, while RN allocates RBs from set $R B_{r}$ to R-UEs to meet QoS requirements. D-UEs and R-UEs are no longer competitive relationship, and each node schedules its UEs independently. If the traffic of D-UEs is mixed GBR with Non-GBR traffic, then the scheduling priority formula of D-UEs in backhaul links is also expressed as (9). If all the traffics of D-UEs are GBR traffics, the scheduling priority formula is as follows:

$$
Q_{k, n}(t)=\frac{H^{\prime} L_{k}(t)}{\text { Delay }_{k}} \cdot \frac{r_{k, n}(t)}{\overline{\mathrm{R}}_{k}(t)} \cdot(10-P(n))
$$

For R-UEs, the set of delay constraint is different from set in access links as (13), the rest of delay threshold of R-UEs depends on data packet transmission delay in access links, so the time delay threshold of different R-UEs is different. Thus, in backhaul sub-frames, the scheduling priority formula of R-UEs is:

$$
Q_{k, \text { Delay }}(t)=\frac{H L_{k}(t)}{\text { Delay }_{k}-H O L_{k}(t)}
$$

\section{Simulation Results and Analysis}

A system level simulation is implemented in MATLAB and performance of the proposed QoS-aware SPRC scheduling scheme is greater than that of the existing scheme TH-MLWDF and TFQoS. The simulation in TD-LTE-A relay system can be divided into three parts: system initialization, data transmission and result statistics. Seven three-sectored cells with one RN per sector are set and the inter-site distance is $500 \mathrm{~m}$. The system carrier frequency is $2 \mathrm{GHz}$ and system bandwidth is $5 \mathrm{MHz}$. The eNB transmit power is set to $46 \mathrm{dBm}$ and the RN transmit power is $30 \mathrm{dBm}$. ITU-PedA channel is used as the channel model. In this simulation, two kinds of GBR video traffic 
and one kinds of Non-GBR traffic is set.

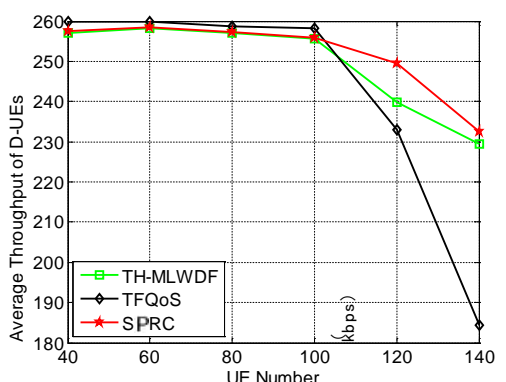

Fig. 4 Throughput of D-UEs vs UE number

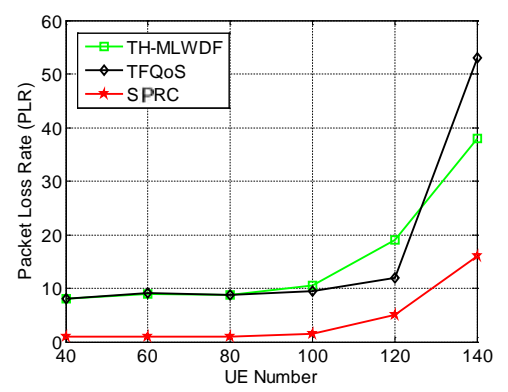

Fig. 7 PLR vs UE number

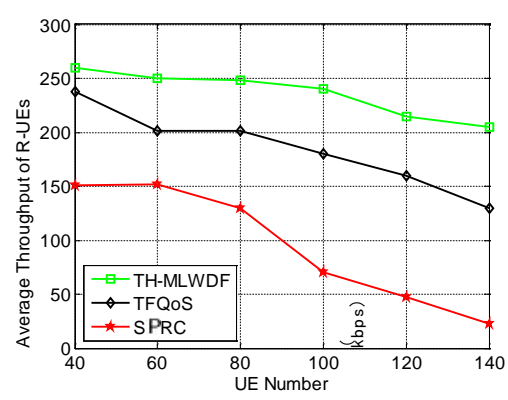

Fig. 5 Throughput of R-UEs vs UE number

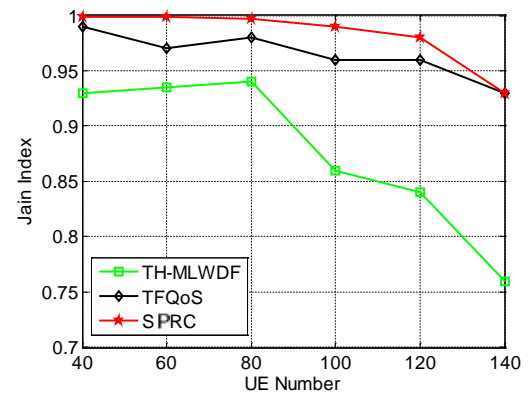

Fig.8 Jian's fairness index vs UE number

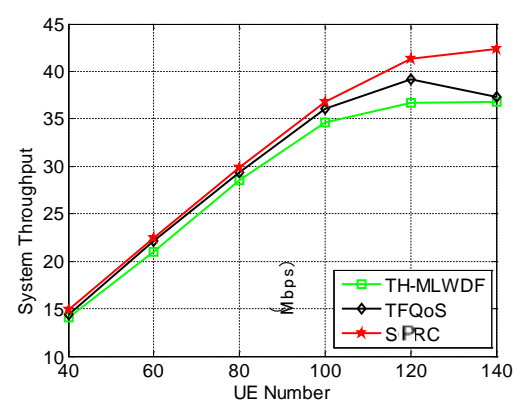

Fig. 6 System throughput vs UE number

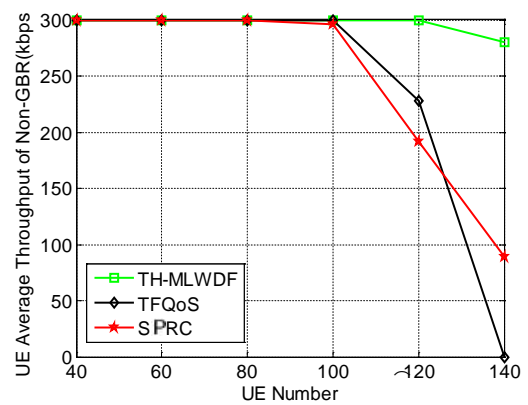

Fig. 9 Throughput of Non-GBR vs UE number

Fig. 4 show the throughput of D-UEs in different algorithms with different UE number. When UE number is within 100, D-UEs' average throughput remain around 259kbps smoothly in three algorithms. However, with the further increase of UE number, D-UEs' average throughput is falling, with the maximum drop in TFQoS algorithm and minimum drop in proposed SPRC algorithm. Fig. 5 presents the R-UEs' average throughput with different UE number. With the increase of UE number, throughput in three algorithms falls slowly. The result shows that the performance of SPPC algorithm is $25 \%$ better than that of TFQoS, and TH-MLWDF algorithm has a bad performance due to its lack of consideration of R-UEs.

Fig. 6 is system throughput comparison chart. When UE number is 40, the performance of system throughput in three algorithms is close. With the increase of UE number, system throughput in three algorithms increases, where the advantages of SPRC algorithm proposed in this paper appeared gradually, compared with the other two algorithms. When UE number reaches 140, SPRC algorithm throughput has a $20 \%$ improvement than the other two algorithms.

Fig. 7 analyzes PLR of all UEs in three algorithms. It is obvious that PLR in SPRC algorithm under the condition of all the different UEs are lower than TH-MLWDF and TFQoS algorithm. Most of discarded packets belong to R-UEs due to R-UEs' more strict requirement of time delay. The priority defined in TH-MLWDF algorithm doesn't consider the specificity of the R-UEs, and it doesn't dynamically adjust the delay threshold of R-UEs in backhaul sub-frames, resulting in poor sensitivity to delay. As for TFQoS algorithm, the proportion in GBR priority factor and time delay priority factor is unreasonable. The priority in SPRC algorithm adds GBR priority factor reasonably and dynamically adjust the delay of R-UEs in backhaul sub-frames to ensure R-UEs' performance.

In Fig. 8, fairness among all UEs in three algorithms is illustrated. Jain's fairness index in SPRC and TFQoS algorithm are higher than 0.95, and that of TH-MLWDF algorithm reaches above 0.9 under abundant system wireless resource, but with the further increase of UE number, fairness among UEs falls down due to the lack of resources.

Fig. 9 gives average throughput comparison chart of UEs who use Non-GBR traffic. TH-MLWDF algorithm does not set different priority for different traffic type, so that it can't accurately distinguish traffic requirements of various UEs, when the traffic are combined with GBR and Non-GBR traffic, resulting in fewer resources are allocated to GBR traffic. SPRC and TFQoS algorithm considers traffic priorities. However, when UE number reaches 140, the throughput of 
GBR traffic in TFQoS algorithm is 0. In other words, it gives GBR traffic the absolute high priority, completely sacrificing the performance of Non-GBR traffic. As for SPRC algorithm, UEs whose traffic is Non-GBR with better channel condition also can be scheduled.

\section{Conclusion}

For the sake of the demands for the transmission of multimedia applications and meeting various QoS requirements of different traffics, we propose a QoS-aware scheduling algorithm SPRC, and compared it with TH-MLWDF and TFQoS. The simulation results show the superiority of SPRC. From the point of view in UE percentage of meeting GBR requirement, SPRC algorithm can well ensure GBR traffic. In terms of PLR, SPRC algorithm has lower PLR and can satisfy UEs' time delay requirements commendably. As for the throughput performance in SPRC algorithm, both R-UEs and D-UEs' throughput can be improved. As for fairness, SPRC algorithm can guarantee the fairness of UEs with various traffics.

\section{Acknowledgement}

This work was supported by the National Basis Research Program of China (973 Program) under Grant No. 2013CB329003, and National Natural Science Foundation of China under Grant No. 61301100 .

\section{References}

[1] 3GPP TS 36.814, “Evolved Universal Terrestrial Radio Access (E-UTRA): Further advancements for E-UTRA physical layer aspects,” V9.0.0, 2010.

[2] $\mathrm{Q} \mathrm{Li}, \mathrm{RQ} \mathrm{Hu}, \mathrm{Y}$ Qian and $\mathrm{G} \mathrm{Wu}$, "A proportional fair radio resource allocation for heterogeneous cellular networks with relays," IEEE Global Communications Conference (GLOBECOM), 2012: 5457-5463.

[3] Mohamed Salem, Abdulkareem Adinoyi, Mahmudur Rahman, Halim Yanikomeroglu, David Falconer and Young-Doo Kim, "Fairness-aware radio resource management in downlink OFDMA cellular relay networks,” IEEE Transactions on Wireless Communications, 2010, 9(5): 1628-1639.

[4] M Salem, A Adinoyi, H Yanikomeroglu and D Falconer, "Fair Resource Allocation Toward Ubiquitous Coverage in OFDMA-Based Cellular Relay Networks With Asymmetric Traffic,” IEEE Transactions on Vehicular Technology, 2011, 60(5): 2280-2292.

[5] Piro G, Grieco LA, Boggia G. and Camarda P., "QoS provisioning in LTE-A networks with relay nodes,” 2012 IFIP Wireless Days. IEEE, 2012:1-3.

[6] X Lei, XR Chen, RQ Hu and G Wu, "Mobile association for heterogeneous wireless relay networks with statistical QoS guarantees,” Globecom Workshops (GC Wkshps) IEEE, 2013: 4933-4939.

[7] TMD Moraes, AA Gonzalez, MD Nisar and E Seidel, "QoS-aware Resource Allocation for In-band Relaying in LTE-Advanced,” The Eighth International Conference on Wireless and Mobile Communications (ICWMC 2012). IEEE, 2012: 195-201.

[8] F Afroz, K Sandrasegaran and P Ghosal, "Performance analysis of PF, M-LWDF and EXP/PF packet scheduling algorithms in 3GPP LTE downlink," Telecommunication Networks and Applications Conference (ATNAC), 2014 Australasian. IEEE, 2014:87-92.

[9] G Piro, LA Grieco, G Boggia and P Camarda, "QoS provisioning in LTE-A networks with relay nodes,” 2012 IFIP Wireless Days. IEEE, 2012:1-3.

[10]G. Piro, LA Grieco, G Boggia, R Fortuna and P Camarda, “Two-level Downlink Scheduling for Real-Time Multimedia Services in LTE Networks,” IEEE Trans. Multimedia, vol. 13, no. 5, pp. 1052 -1065, Oct. 2011. 
[11]F Capozzi, G Piro, LA Grieco, G Boggia and P Camarda, "Downlink Packet Scheduling in LTE Cellular Networks: Key Design Issues and a Survey,” IEEE Communication Surveys \& Tutorials, vol. 15, no. 2, pp.678-700, 2013. 